\title{
Nonlinear X-Wave Ultrasound Imaging of Acoustic Biomolecules
}

\author{
David Maresca, ${ }^{1, * \dagger}$ Daniel P. Sawyer, ${ }^{2, *}$ Guillaume Renaud, ${ }^{3}$ Audrey Lee-Gosselin, ${ }^{1}$ and Mikhail G. Shapiro ${ }^{1, *}$ \\ ${ }^{1}$ Division of Chemistry and Chemical Engineering, California Institute of Technology, \\ Pasadena, California 91125, USA \\ ${ }^{2}$ Division of Biology and Biological Engineering, California Institute of Technology, \\ Pasadena, California 91125, USA \\ ${ }^{3}$ Laboratoire d'Imagerie Biomédicale, \\ Sorbonne Université-CNRS UMR7371-INSERM U1146, Paris, 75006, France
}

(Received 6 April 2018; revised manuscript received 23 July 2018; published 4 October 2018)

The basic physics of sound waves enables ultrasound to visualize biological tissues with high spatial and temporal resolution. Recently, this capability was enhanced with the development of acoustic biomolecules_-proteins with physical properties enabling them to scatter sound. The expression of these unique air-filled proteins, known as gas vesicles (GVs), in cells allows ultrasound to image cellular functions such as gene expression in vivo, providing ultrasound with its analog of optical fluorescent proteins. Acoustical methods for the in vivo detection of GVs are now required to maximize the impact of this technology in biology and medicine. We previously engineered GVs exhibiting a nonlinear scattering behavior in response to acoustic pressures above $300 \mathrm{kPa}$ and showed that amplitudemodulated (AM) ultrasound pulse sequences that excite both the linear and nonlinear GV scattering regimes were highly effective at distinguishing GVs from linear scatterers like soft biological tissues. Unfortunately, the in vivo specificity of AM ultrasound imaging is systematically compromised by the nonlinearity added by the GVs to propagating waves, resulting in strong image artifacts from linear scatterers downstream of GV inclusions. To address this issue, we present an imaging paradigm, crossamplitude modulation (xAM), which relies on cross-propagating plane-wave transmissions of finite aperture $\mathrm{X}$ waves to achieve quasi-artifact-free in vivo imaging of GVs. The XAM method derives from counterpropagating wave interaction theory, which predicts that, in media exhibiting quadratic elastic nonlinearity like biological tissue, the nonlinear interaction of counterpropagating acoustic waves is inefficient. By transmitting cross-propagating plane waves, we minimize cumulative nonlinear interaction effects due to collinear wave propagation while generating a transient wave-amplitude modulation at the two plane waves' intersection. In both simulations and experiments, we show that residual xAM nonlinearity due to wave propagation decreases as the plane-wave cross-propagation angle increases. We demonstrate in tissue-mimicking phantoms that imaging artifacts distal to GV inclusions decrease as the plane-wave cross-propagation angle opens, nearing complete extinction at angles above 16.5 degrees. Finally, we demonstrate that XAM enables highly specific in vivo imaging of GVs located in the gastrointestinal tract, a target of prime interest for future cellular imaging. These results advance the physical facet of the emerging field of biomolecular ultrasound and are also relevant to synthetic ultrasound contrast agents.

DOI: 10.1103/PhysRevX.8.041002

\footnotetext{
*These authors contributed equally to this work.

${ }^{\dagger}$ Corresponding author.

dmaresca@caltech.edu

${ }^{\ddagger}$ Corresponding author. mikhail@caltech.edu
}

Published by the American Physical Society under the terms of the Creative Commons Attribution 4.0 International license. Further distribution of this work must maintain attribution to the author(s) and the published article's title, journal citation, and DOI.
Subject Areas: Acoustics, Biological Physics, Nonlinear Dynamics

\section{INTRODUCTION}

Green fluorescent protein (GFP) and its analogs serve as irreplaceable tools allowing biologists to visualize gene expression and other cellular processes using optical microscopes [1]. However, the microstructure of biological tissues restricts a photon's transport mean free path to about $1 \mathrm{~mm}$ [2], limiting in vivo optical imaging applications. In contrast, the physics of ultrasonic waves allows them to propagate centimeters deep into biological tissues without losing their coherence and enables tissue scanning at the 
(a) Nonlinear Wave Propagation - at source - at distance D1 - at distance D2 > D1

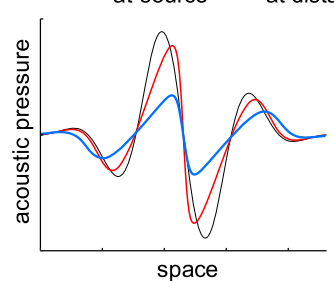

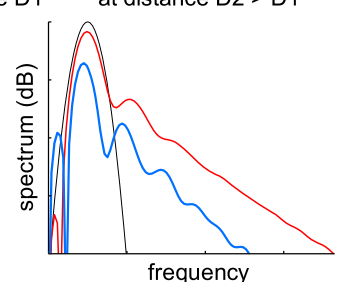

frequency (b)

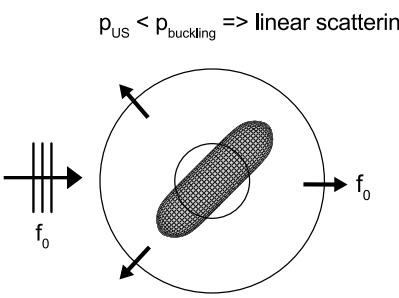

Nonlinear Scattering of Gas Vesicles

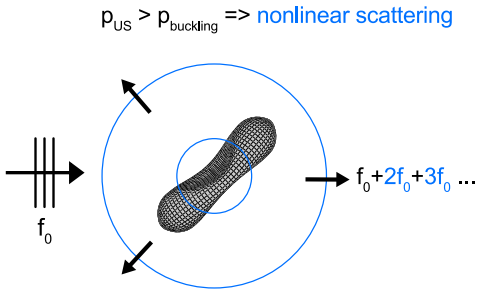

FIG. 1. Sketch of the two nonlinear phenomena that take place while imaging a biological medium containing acoustic biomolecules. (a) Propagation history of a single plane wave: Nonlinear frequency components accumulate with depth as the wave propagates through tissue before being attenuated. This phenomenon, amplified during the near-collinear interaction of two wavefronts, leads to nonlinear propagation artifacts distal to GV inclusions. (b) Nonlinear scattering behavior of GVs insonified above their buckling pressure, enabling their detection with an AM code.

organ scale. Very recently, the first acoustic biomolecules for ultrasound, analogous to GFP for optics, were developed [3] based on a unique class of air-filled protein nanostructures called gas vesicles, or GVs [4], making it possible to use ultrasound to visualize the function of cells deep inside tissues.

To maximize the impact of acoustic biomolecules in biology and medicine, physical methods are needed to discriminate GV scattering from tissue scattering, analogous to previous developments in the imaging of synthetic microbubble contrast agents [5-11]. GVs are air-filled nanocompartments with dimensions on the order of $200 \mathrm{~nm}$, enclosed by a rigid 2-nm-thick protein shell $[12,13]$. In 2014, it was reported that GVs could be imaged with ultrasound [4], and it was recently shown that gene clusters encoding GVs could be expressed heterologously in engineered cells and serve as acoustic reporter genes [3]. While most natural GVs behave as linear ultrasound scatters, Lakshmanan et al. [14] engineered harmonic GV variants (hGVs) that buckle and scatter higher harmonics at acoustic pressures above $320 \mathrm{kPa}$, corresponding to a mechanical index of 0.08 , well below the FDA safety requirement of 1.9 [15]. Amplitude modulation (AM) ultrasound pulse sequences emerged as logical candidates to exploit dissimilar hGV responses below and above buckling and enabled nonlinear imaging of hGVs in vitro, in ovo, and in vivo [15]. In AM, backscattered echoes of two half-amplitude transmissions are digitally subtracted from echoes of a third, fullamplitude transmission. The full-amplitude transmission creates pressures above the hGV buckling threshold, triggering hGV harmonic scattering, while the half-amplitude transmissions create pressures below hGV buckling and trigger $\mathrm{hGV}$ linear scattering. This scattering response difference in hGV echoes persists after the subtraction, while linear echoes from surrounding tissue scale in amplitude and are canceled. However, we observed that the in vivo specificity of AM imaging of hGVs is systematically compromised by nonlinear wave propagation artifacts that lead to the misclassification of biological tissue as hGVs [15].
In a highly nonlinear medium such as buckling hGVs or resonant microbubbles (nonlinearity parameter B/A 2 orders of magnitude higher than tissue) [16], ultrasonic waves experience amplitude-dependent attenuation and amplitude-dependent speed of sound [17,18]. In consequence, the high-amplitude pulse of the AM sequence gets distorted in proportions that do not scale linearly with the low-amplitude pulse of the sequence. Ultrasonic waves carry that distortion as they travel forward in the medium. We hypothesize that this phenomenon, reported in microbubble inclusions [19], is also the cause of nonlinear artifacts distal to $\mathrm{GV}$ inclusions.

It is established that, in a medium exhibiting quadratic elastic nonlinearity, the interaction of two ultrasonic waves propagating in the same direction exhibits a cumulative nonlinear interaction [20]. With a conventional AM pulse sequence, images are reconstructed line by line along the wave propagation direction. The highamplitude pulse of the sequence can be seen as the sum of two pulses of half amplitude that copropagate and are subject to cumulative nonlinear interaction effects. It is less well known that the nonlinear interaction of ultrasonic waves propagating in opposite directions is inefficient [21]. In a pioneering proof of concept, Renaud et al. [22] showed that a pair of subwavelength elements of an ultrasound transducer array could be used to transmit circular wave pulses that are quasi-counterpropagative in the near field of the array, allowing them to minimize nonlinear distortion while generating a twofold higher amplitude at the moment of their intersection. Here, we show that propagation nonlinearity [Fig. 1(a)] can be efficiently minimized with a noncollinear plane-wave transmission paradigm, which we call cross-amplitude modulation, or xAM, while allowing depth-invariant, nonlinear imaging of acoustic biomolecules [Fig. 1(b)]. We demonstrate in simulations and experiments that plane-wave cross-propagation prevents cumulative distortion of the AM wave code, suppresses nonlinear propagation artifacts distal to highly nonlinear hGV inclusions, and enables highly specific in vivo nonlinear ultrasound imaging of hGVs in mice. 


\section{NONLINEAR INTERACTION OF CROSS-PROPAGATING PLANE WAVES}

A. Cross-amplitude modulation sequence

Cross-amplitude modulation is a biomolecular ultrasound imaging paradigm that aims at minimizing wave propagation-related harmonics [23] using propagation symmetry considerations while capturing local acoustic biomolecules' harmonics to ensure their specific in vivo detection. Considering an N-element aperture of a linear array of ultrasonic transducers, the XAM sequence consists in (1) using the elements 1 to $N / 2$ to transmit a tilted plane wave at an angle $\theta$ with respect to the array [Fig. 2(a)], (2) using the elements $N / 2+1$ to $\mathrm{N}$ to transmit a symmetric plane wave at an angle $\theta$ with the array [Fig. 2(b)], and (3) transmitting the previous two plane waves simultaneously [Fig. 2(c)]. The two cross-propagating waves depicted in Fig. 2(c) interact along the virtual bisector that separates the two half-apertures. Particles of the insonified medium that are located along the bisector experience the same wave amplitude for steps (1) and (2), and a double wave amplitude for step (3), as seen in Fig. 2(d). This axisymmetric pulse sequence creates an AM code along the bisector that separates the two $N / 2$ subapertures.

Our working hypothesis is that a cumulative nonlinear plane-wave interaction arising during wave propagation decreases as the cross-propagation angle $\theta$ increases. We therefore start by determining whether minimal AM code distortion could be achieved with XAM in a weakly nonlinear homogeneous and isotropic medium. To do so, we first evaluate the XAM signal cancellation during plane-wave propagation in water using two-dimensional time-domain numerical simulations (see Appendix A) [24]. Keeping the experimental realization of the XAM sequence in mind, we assess the directivity of individual elements of our transducer array [25] and set the maximal angle $\theta$ to $21^{\circ}$ based on the array $-3 \mathrm{~dB}$ directivity bandwidth (see Appendix A, Fig. 7). The simulation result displayed in Fig. 2(d) shows that, for a xAM sequence of angle $\theta=18^{\circ}$ at a depth of $3.6 \mathrm{~mm}$, the residual peak wave amplitude is reduced by 4 orders of magnitude to $0.02 \%$ of the cross-propagating plane-wave peak amplitude $(0.13 \mathrm{kPa}$ compared to $747 \mathrm{kPa}$, respectively). As a comparison, a high-end commercial scanner provides an AM residual of the order of $0.5 \%$.

Cross-propagating plane waves, or $\mathrm{X}$ waves, intersect with each other along a finite geometric distance that defines the depth of field $Z_{X}$ of the XAM sequence,

$$
Z_{X}=\frac{A}{2} \cot \theta
$$

where $A$ is the full aperture used for the $\mathrm{X}$-wave transmission. For an angle $\theta=18^{\circ}$ and an aperture $A=6.5 \mathrm{~mm}$, $Z_{X}$ equals $10 \mathrm{~mm}$, which would, for example, enable scanning of a full mouse brain. Note that beyond $Z_{X}$, images could be reconstructed further down thanks to the diffraction of the wave-front edges using spherical delay laws analogous to those of Renaud et al. [22].

We further assess the significance of nonlinear effects accumulating during plane-wave propagation as a function of $\theta$ [Figs. 3(a) and 3(b)]. At low $\theta$ angles, which correspond to quasi-copropagation, residual AM nonlinearity clearly accumulates with depth [see Fig. 3(a), $\theta=0^{\circ}$ and $5^{\circ}$ ]. In particular, for plane-wave propagating in water with a peak amplitude of $400 \mathrm{kPa}$ and at an angle $\theta=0^{\circ}$ (collinear propagation case), the residual AM peak amplitude reaches $13.5 \mathrm{kPa}$ at a distance $z=8 \mathrm{~mm}$ [Fig. 3(a)]. As $\theta$ increases, the residual AM nonlinearity is significantly reduced,

$\begin{array}{lll}\text { (a) Transmit event } 1 \quad \text { (b) } & \end{array}$

(b)

Transmit event 2

(c) Transmit event 3

(d)
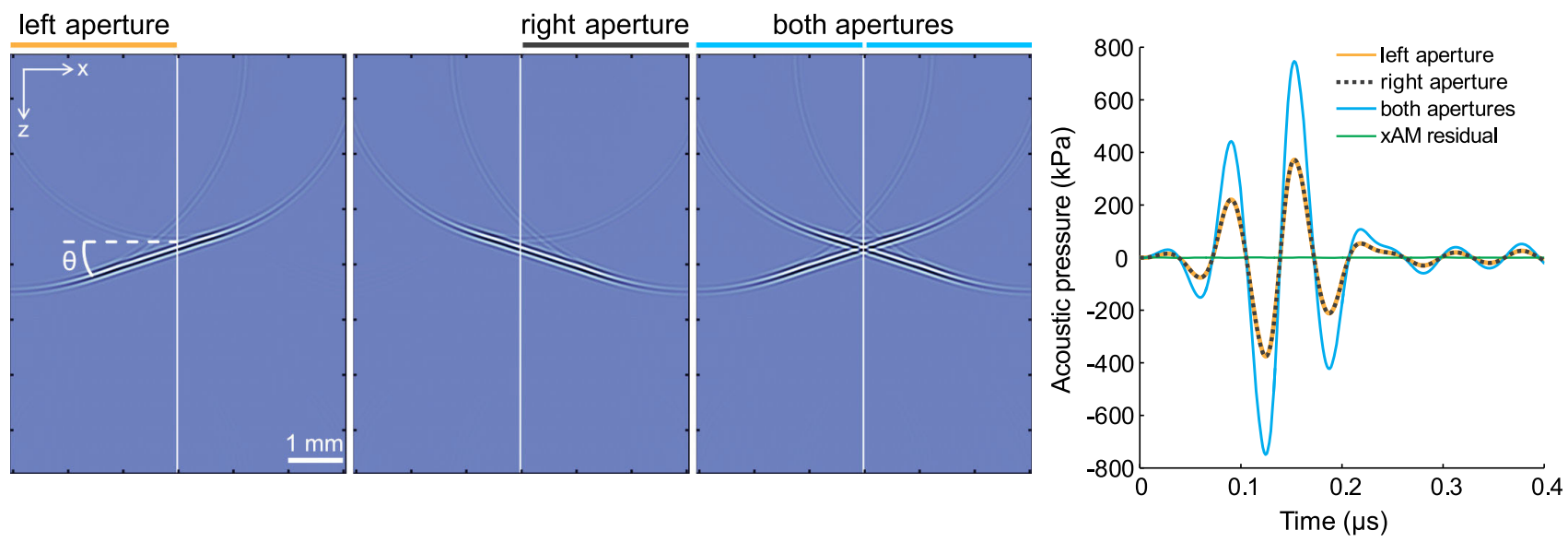

FIG. 2. Simulation of the XAM sequence for $\theta=18^{\circ}$ in a homogeneous and isotropic water medium. (a) Half-aperture plane-wave transmission at an $18^{\circ}$ angle with respect to the transducer array. (b) Axisymmetric half-aperture plane-wave transmission at an $18^{\circ}$ angle with the other half of the array. (c) Cross-propagating plane-wave transmission at an $18^{\circ}$ angle using both half-apertures. (d) Simulated waveforms at the bisector intersection for $z=3.6 \mathrm{~mm}$. The cross-propagating plane-wave peak positive pressure is $747 \mathrm{kPa}$ (blue curve), while the residue peak positive pressure (green curve) is $0.13 \mathrm{kPa}$, or $0.02 \%$ of the cross-propagating plane-wave peak positive pressure. 
(a)

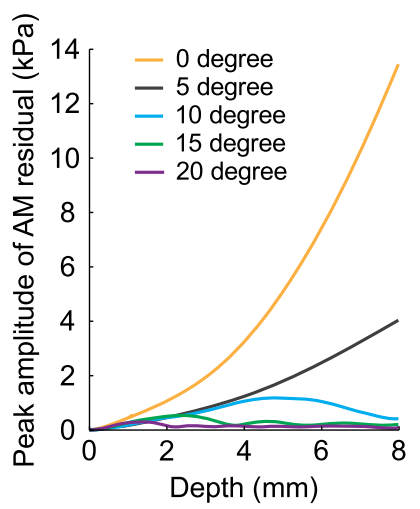

(b)

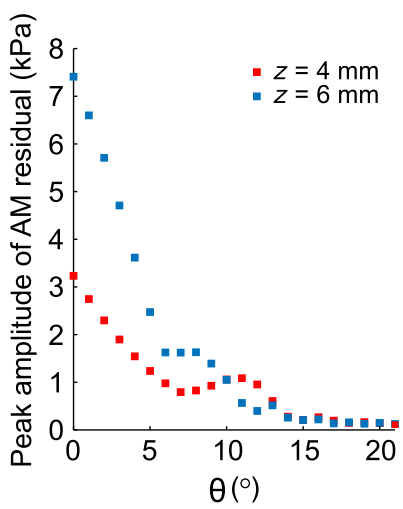

FIG. 3. Simulation of nonlinear plane-wave interaction as a function of the cross-propagation angle $\theta$. (a) Peak positive pressure of the XAM residual as a function of depth for five crosspropagation angles. (b) Peak positive pressure of the xAM residual as a function of $\theta$ at depths $z$ equal to $4 \mathrm{~mm}$ and $6 \mathrm{~mm}$.

reaching a noncumulative $0.3 \mathrm{kPa}$ peak pressure at an angle $\theta=20^{\circ}$ [Fig. 3(a)]. Figure 3(b) reports the peak amplitude of the AM residue as a function of $\theta$ at the distances $z=4 \mathrm{~mm}$ and $z=6 \mathrm{~mm}$ from the array. Data reported in Figs. 3(a) and 3(b) were obtained using a constant transmit peak pressure at $4 \mathrm{~mm}$ equal to $400 \mathrm{kPa}$. The simulations show that the amplitude of the XAM residue drops rapidly as $\theta$ increases and converges below a threshold of $0.2 \mathrm{kPa}$ for $\theta>15^{\circ}$. These results support our hypothesis that a nonlinear planewave interaction becomes less efficient as $\theta$ increases, and we predict that in a weakly nonlinear homogeneous and isotropic medium such as water (attenuation equal to $0.002 \mathrm{~dB} / \mathrm{MHz}^{2} \mathrm{~cm}$ ), one can expect an AM code showing minimal wave distortion due to propagation at cross-propagation angles above $15^{\circ}$.
While a complete analytical treatment of this phenomenon has not been developed, some intuition can be obtained from the work of Hamilton et al. [26,27], who reported that the noncollinear nonlinear interaction of two plane-wave fields results in an asynchronous interaction that generates a sum frequency wave whose amplitude oscillates with a spatial period of $2 \pi / 2 k(1-\cos \theta)$, with $k$ being the wave vector of the plane waves. As the angle between the two waves increases, the phase mismatch increases, the spatial period of the nonlinear pressure field decreases, and the nonlinear interaction becomes less efficient. The same phenomenon is observed in our simulations: As the angle increases, the spatial period of oscillation and the amplitude of the nonlinear pressure field decrease [see 10-, 15-, and 20-degree lines in Fig. 3(a)]. The nonlinear pressure field is also expected to increase or decrease with the nonlinearity of the medium, as characterized by its shock length. With these considerations supporting the plausibility of our simulation results, we proceed to implement the xAM concept experimentally. A full analytical treatment of this problem will be described in future work.

\section{B. Experimental reduction of residual XAM nonlinearity as a function of $\boldsymbol{\theta}$}

To experimentally assess the ability of higher $\theta$ xAM pulses to minimize nonlinearity, we implement the xAM imaging sequence and beamforming on a programmable ultrasound system with a 128-element linear array (see Appendix A) and measure the peak residual AM signal of a subwavelength linear scatterer immersed in water. This configuration enables the assessment of the nonlinearity captured by the AM sequence that is solely due to wave propagation in a typical quasi-incompressible medium (water (a)

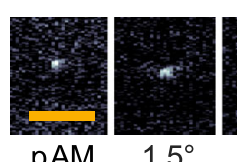

$\mathrm{pAM}$

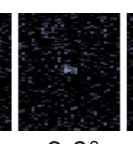

$3.0^{\circ}$

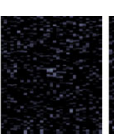

$4.5^{\circ}$



$6.0^{\circ}$

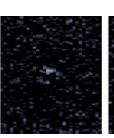

$7.5^{\circ}$

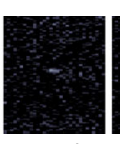

$9.0^{\circ}$

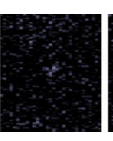

$10.5^{\circ}$

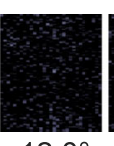

$12.0^{\circ}$

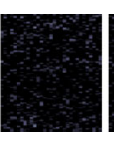

$13.5^{\circ}$

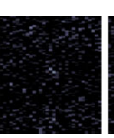

$15.0^{\circ}$

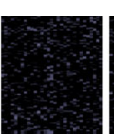

$16.5^{\circ}$

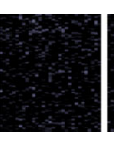

$18.0^{\circ}$

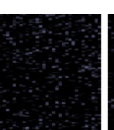

$19.5^{\circ}$

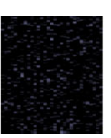

$21.0^{\circ}$

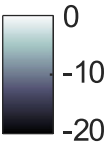

$(\mathrm{dB})$

(b)

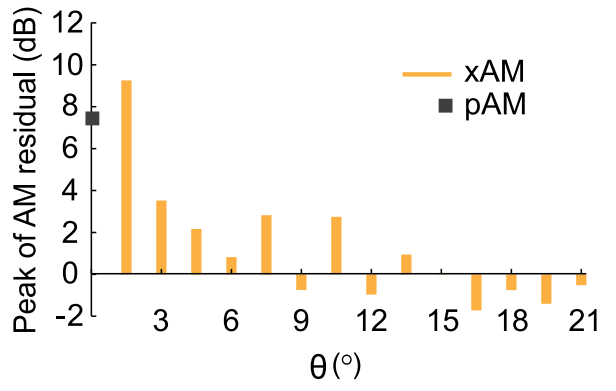

FIG. 4. xAM images of the cross section of a subwavelength nickel wire as a function of the cross-propagation angle $\theta$. (a) Images reconstructed from the three component transmissions of the pAM code and xAM code at angles ranging from $1.5^{\circ}$ to $21^{\circ}$. The wire is positioned at a depth of $4 \mathrm{~mm}$. Each image depth ranges from $3.0 \mathrm{~mm}$ to $4.5 \mathrm{~mm}$ and each width from $-1.5 \mathrm{~mm}$ to $1.5 \mathrm{~mm}$. Scale bar: $1 \mathrm{~mm}$. (b) Peak AM residual signal as a function of the XAM sequence angle $\theta$. The xAM signals are labeled in orange; the pAM signal is labeled with a gray square symbol. Values in $\mathrm{dB}$ represent the peak value of the residual signal relative to the peak value of the noise. 



(b)

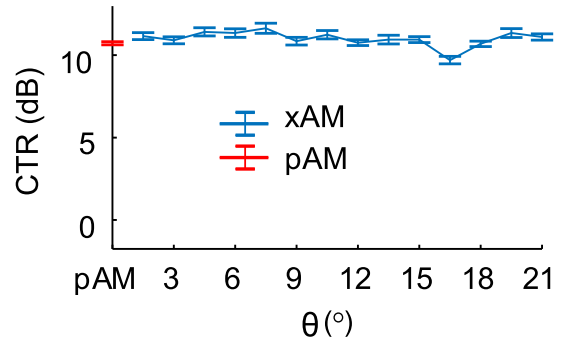

(c)

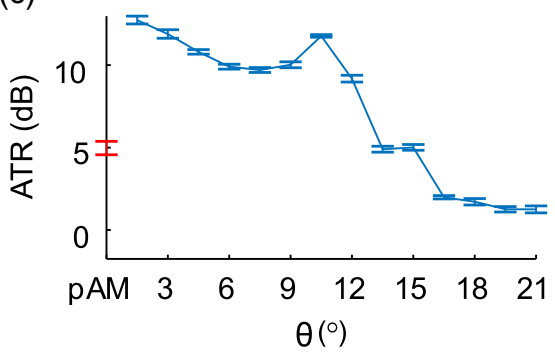

(d)

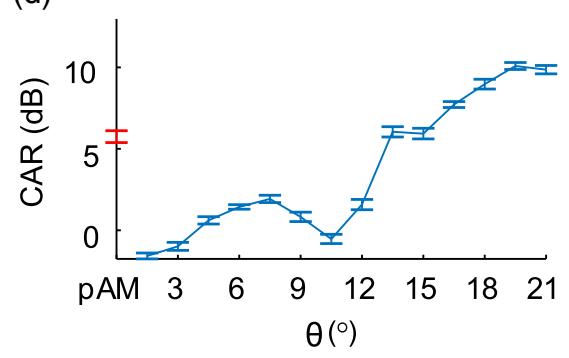

FIG. 5. In vitro pAM and XAM images of a hGV inclusion in a tissue-mimicking phantom. (a) Left panel: Schematic of the phantom configuration. The linearly scattering tissue-mimicking medium is shown in gray, the hGV inclusion in blue, and the anechoic agar-filled inclusion in black. ROIs are shown for contrast (C), tissue (T), and artifact (A) quantification. Right panel: Set of pAM and xAM images of a representative well positioned at $z=4 \mathrm{~mm}$. Separate images spanning depths of $3 \mathrm{~mm}$ to $9 \mathrm{~mm}$ are concatenated. Scale bar: $1 \mathrm{~mm}$. White dotted line: $Z_{X}$ at $\theta=21^{\circ}$. (b) CTR as a function of $\theta$. (c) ATR as a function of $\theta$. (d) CAR as a function of $\theta$. $N=6$. Error bars: standard error of the mean (SEM).

serves as a first-approximation model for sound-wave propagation in soft biological tissues). A $10 \mu \mathrm{m}$ diameter nickel wire is placed perpendicular to the imaging plane in phosphate buffer saline (PBS) at a depth of $4 \mathrm{~mm}$. The wire is imaged using $x A M$ at $\theta$ values ranging from $1.5^{\circ}$ to $21^{\circ}$ [Fig. 4(a)]. We measure the AM residual for each angle as the peak value of the beamformed radio-frequency (rf) data. The data show the same trend predicted by the simulations, with residual nonlinearity decreasing sharply with wider angles [Fig. 4(b)]. To compare these results with a conventional AM imaging sequence, we implement a standard parabolic amplitude modulation (pAM) code, in which half-amplitude transmissions are achieved by silencing the even or odd transmitting elements of the array, and imaging lines are reconstructed along the wave propagation direction [15]. As of $\theta=3^{\circ}$, xAM significantly outperforms pAM in reducing the residual AM signal [Fig. 4(b)]. We measure the axial and lateral resolution of XAM using the full-width halfmaximum (FWHM) of the point-spread function (PSF) along the respective directions. The mean axial resolution is $117 \mu \mathrm{m} \pm 16 \mu \mathrm{m}$, and the mean lateral resolution is $381 \mu \mathrm{m} \pm 42 \mu \mathrm{m}$, with values remaining constant across angles. The axial resolution of pAM is $103 \mu \mathrm{m}$, and the lateral resolution is $250 \mu \mathrm{m}$.

\section{CROSS-AMPLITUDE MODULATION IMAGING OF ACOUSTIC BIOMOLECULES}

\section{A. Angle-dependent XAM reduction of nonlinear propagation artifacts distal to $\mathbf{G V}$ inclusions}

The XAM sequence was developed to detect hGVs with high specificity. The peak positive pressure of the single tilted plane waves excites the hGVs in the linear scattering regime, while the doubled $\mathrm{X}$-wave intersection amplitude excites the hGVs in the nonlinear scattering regime. By summing the echoes from the two plane-wave transmissions and then subtracting them from the echoes of the $\mathrm{X}$-wave transmissions, we solely retrieve nonzero differential GV signals, while the echoes of surrounding linear scatterers cancel. To evaluate the effectiveness of xAM in reducing the nonlinear propagation artifact, we embed a 2-mm-wide cylindrical inclusion of hGVs in agar (at a concentration of $256 \mathrm{pM}$; see Appendix A for GV preparation) in a tissue-mimicking phantom consisting of agar and $3-\mu \mathrm{m}$ aluminum oxide particles (a model linear scatterer). A second inclusion filled with a scatterer-free PBS/agar mixture is positioned $1 \mathrm{~mm}$ below the GVs [see schematic, Fig 5(a)]. We image the phantom using the same sequence parameters used for the subwavelength scatterer measurements, with the top of the hGV inclusion positioned at $4 \mathrm{~mm}$ [Fig. 5(a)] since $\mathrm{X}$ waves provide extended depths of fields compared to parabolic beams [28].

With parabolic and low- $\theta$ xAM pulses, we observe a significant nonlinear propagation artifact distal to $\mathrm{hGV}$ inclusions [Figs. 5(a) and 5(b)], confirming the high nonlinearity of hGV-filled media. The pAM and xAM images were quantified in terms of contrast-to-tissue ratios (CTR), contrast-to-artifact ratios (CAR), and artifact-totissue ratios (ATR) [Figs. 5(b)-5(d)]. For pAM and low- $\theta$ angles, the artifact intensity is on par with or above the hGV inclusion intensity (e.g., for $\theta=1.5^{\circ}, \mathrm{CAR}=-1.6 \mathrm{~dB}$ at $z=4 \mathrm{~mm}$ ), highlighting the specificity issue posed by collinear AM imaging. For angles above $15^{\circ}$, xAM 
(a)

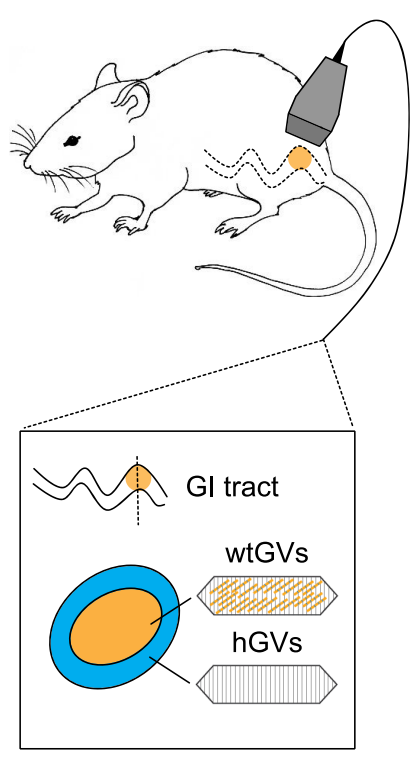

(b) pBMode

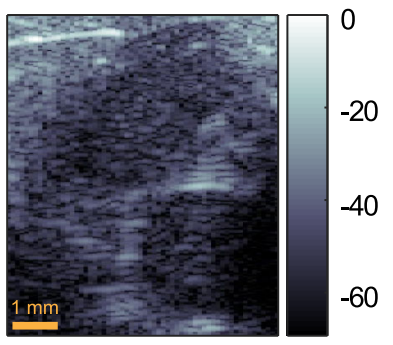

(c) pAM

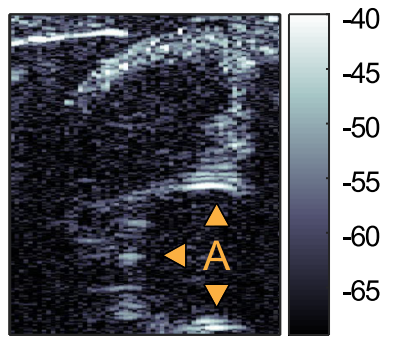

(d) $\quad x B M o d e$



(e) $\quad x A M$

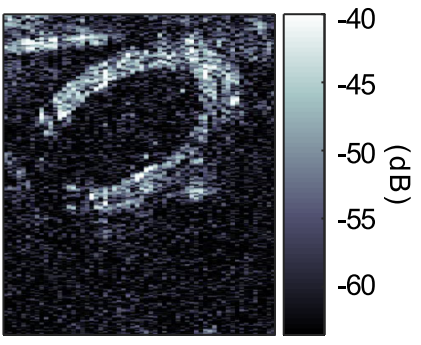

(f)
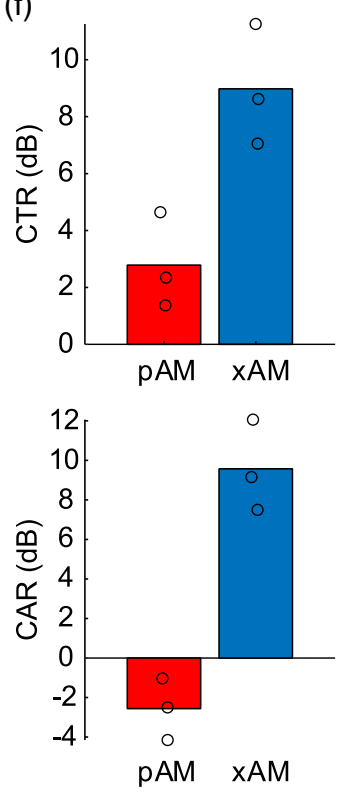

FIG. 6. In vivo pAM and XAM imaging of acoustic biomolecules. (a) Schematic of the experiment. A concentric mixture of nonlinearly scattering hGVs and linearly scattering wtGVs is injected in a mouse gastrointestinal (GI) tract and imaged with pAM and xAM. (b) pBMode image, focus $=4 \mathrm{~mm}, f$ - number $=2.0$. (c) pAM image, arrows point to the artifact (A). (d) xBMode, $\theta=19.5^{\circ}$. (e) XAM image. The pAM and XAM dynamic ranges are displayed relative to their respective BMode ranges. All image depths range from $z=2 \mathrm{~mm}$ to $Z_{X}=9.2 \mathrm{~mm}$. (f) Comparison of XAM and pAM in terms of mean CTR and CAR. $N=3$. ROIs for CTR and CAR measurements are reported in Fig. 8, Appendix A.

produces images with a clear reduction in artifact signal while maintaining full contrast in the hGV inclusion. We also observe that, as in the simulation and subwavelength scatter results, the artifact reduction is a nonmonotonic function of $\theta$, with a local jump in the xAM artifact at $10.5^{\circ}$ [Fig. 5(c)]. Overall, these results suggest that XAM provides the highest specificity for hGV signals at angles larger than $15^{\circ}$.

\section{B. In vivo $\mathrm{xAM}$ ultrasound imaging of acoustic biomolecules}

Finally, to test the XAM imaging method in vivo, we inject into the gastrointestinal tract of a mouse a patterned agar-GV mixture that consists of a core of wild-type linearly scattering GVs (wtGVs) surrounded by a circular layer of hGVs [Fig. 6(a)]. We image the mouse abdomen using xAM at $\theta=19.5^{\circ}$, which yields the highest contrastto-artifact ratio in the phantom experiments, and we compare the results to imaging with pAM with the focus adjusted to $4 \mathrm{~mm}$ and an aperture of 20 elements $(f-$ number $=2.0)$ to align the depth of field with that of the XAM sequence. The parabolic B-mode (pBMode) image, i.e., the conventional anatomical ultrasound image, is sharper than the cross-propagating B-mode (xBMode) image [Figs. 6(b) and 6(d)], which is expected, as X waves generate higher side lobes that reduce image contrast [29]. Only the top of the hGV inclusion is visible in the pAM image due to the narrow depth of field of parabolic beams
[Fig. 6(c)]. The pAM image also contains a large artifact below the inclusion $(\mathrm{CTR}=2.8 \mathrm{~dB}, \mathrm{CAR}=-2.6 \mathrm{~dB})$, parts of which blend with the hGV signal, displaying a potentially misleading distribution of the contrast agent. In contrast, the annular hGV inclusion is almost entirely visible in the XAM image, with little-to-no artifact in the vicinity, and inner and outer contours more clearly delineated [Fig. 6(e)]. The xAM CTR is $9.0 \mathrm{~dB}$, and the xAM CAR is $9.6 \mathrm{~dB}$, demonstrating the superior performance of xAM over pAM in terms of specificity [Fig. 6(f)].

\section{ADVANTAGES, LIMITATIONS, AND OUTLOOK}

Taken together, our results suggest that the XAM ultrasound pulse sequence, based on one $\mathrm{X}$ wave and two tilted plane-wave transmissions, achieves highly specific nonlinear imaging of acoustic biomolecules through wave-amplitude modulation. Two noncollinear plane waves interact to generate a twofold amplitude modulation at their intersection with minimal nonlinear distortion for angles $\theta$ above $15^{\circ}$. This allows retrieval of nonzero differential hGV signals, while the echoes of surrounding linear scatterers cancel and propagation artifacts are reduced to the noise floor level $\left(-10 \mathrm{~dB}\right.$ at $\left.18^{\circ}\right)$. With a $6.4-\mathrm{mm}$ aperture, this technique offers a depth of field suitable for small-animal imaging $\left(10 \mathrm{~mm}\right.$ at $\left.18^{\circ}\right)$. The xAM sequence proves to be robust enough to suppress in vivo artifacts present in pAM while distinguishing engineered nonlinear hGVs from linearly 
scattering wild-type $\mathrm{GV}$ variants in the gastrointestinal tract of a mouse. While this work focuses on introducing and thoroughly characterizing the XAM pulse sequence for use with nonlinear contrast agents, with purified GVs as the model agent, these results are relevant to parallel work being done to apply GVs as functionalized contrast agents and as reporter genes expressed inside cells $[3,14,30]$. The XAM imaging method introduced here thus paves the way for in vivo biomolecular ultrasound studies of molecular and cellular processes based on visualization of acoustic biomolecules [13]. Interestingly, theory and simulations predict that the peak of the cross-propagating plane waves travels at a supersonic velocity, increasing as $\theta$ opens (see Appendix B, Fig. 9), an effect that may be linked to the decreasing nonlinear interaction of the planar wavefronts. Coherent compounding of xAM data acquired at four different angles $\theta$ (see Appendix B, Fig. 10) is also shown to be a way to increase CTR and CAR further.

Potential limitations of this method include its reduced depth of field as the cross-propagating angle increases. Therefore, the method appears to be well suited for ultrasound biomicroscopy, small animal experiments, and superficial examinations in humans. The XAM image depth can be extended beyond the intersection distance of the $\mathrm{X}$ wave by using spherical delay laws reported by Renaud et al. [22], but the quality of nonlinear artifact reduction will decline with depth. Another potential limitation of the method is that it relies on propagation symmetry in a homogeneous and isotropic medium to generate an amplitude-modulated code, and it will therefore be impacted by dissymmetric phase-aberrating media like biological tissues. This issue could be tackled using adaptive wavefront-shaping techniques developed in optics and acoustics [31,32]. In the near future, xAM could be implemented at ultrafast frame rates [33] by relying on 2D arrays of transducers [34] to improve the sensitivity or the temporal resolution of XAM further. Finally, while this study focuses on the use of XAM to image nonlinear acoustic biomolecules, we expect this technology to propagate across as a general solution to the long-standing problem of distal nonlinear propagation artifacts in the field of synthetic microbubble-based ultrasound contrast agents.

\section{ACKNOWLEDGMENTS}

D. M. is supported by the Human Frontier Science Program (Grant No. LT000637/2016). D. S. is supported by the NSF (Grant No. 1745301). This research was funded by the National Institutes of Health (Grant No. EB018975 to M. G.S.). Related research in the Shapiro Laboratory is also supported by the Heritage Medical Research Institute, Burroughs Wellcome Career Award at the Scientific Interface, the Pew Scholarship in the Biomedical Sciences, and the Packard Fellowship for Science and Engineering.
D. M. and D. S. contributed equally to this work.

\section{APPENDIX A: MATERIALS AND METHODS}

\section{1. k-Wave simulations}

We investigate the influence of the transmit angle $\theta$ on the nonlinear interaction between two noncollinear plane waves emitted by two apertures using two-dimensional time-domain numerical simulations (k-Wave version 1.2, [24]). The transmit angle was varied from 1 to 21 degrees (Fig. 3). Transmit delays are calculated to generate a plane wave with the proper angle $\theta$. We simulate wave propagation in a homogeneous and isotropic medium (water) with a configuration corresponding to the setup reported experimentally in Fig. 4. Speed of sound in water is set to $1480 \mathrm{~m} / \mathrm{s}$, the attenuation to $0.002 \mathrm{~dB} / \mathrm{MHz}^{2} \mathrm{~cm}$, and the nonlinear parameter $\mathrm{B} / \mathrm{A}$ to 5 . The size of the domain is $6.4 \mathrm{~mm} \times 8 \mathrm{~mm}$; it is discretized with a step size of $10 \mu \mathrm{m}$. Perfectly matched layers are used to absorb the waves at the edges of the domain. The source broadcasts a short pulse with a center frequency of $15 \mathrm{MHz}$. The acoustic pressure generated by the source is varied so that the peak acoustic pressure generated at 4-mm depth by a single aperture equals $400 \mathrm{kPa}$ for all tested angles. For a given transmit angle, three simulations are required: (1) transmission with the right aperture only, (2) transmission with the left aperture only, and (3) transmission with both apertures. The pressure field is recorded along the segment bisector (between the two transmit apertures). For a given transmit angle, the amplitude modulation scheme is applied to the recorded signals; then, the result is bandpass filtered to reproduce the effect of the limited frequency bandwidth of the transducer with a $100 \%$ relative frequency bandwidth (the cutoff frequencies of the filter are $7.5 \mathrm{MHz}$ and $22.5 \mathrm{MHz}$ ).

\section{Engineering of harmonic acoustic protein nanostructures}

Anabaena GVs are cultured and transferred to sterile separating funnels, and the buoyant cells are allowed to float to the top and separate from the spent media over a $48 \mathrm{~h}$ period. GVs are harvested by hypertonic lysis. Purification is done by repeated centrifugally assisted flotation followed by resuspension. Wild-type Ana GVs are stripped of their outer GvpC layer by treatment with 6-M urea solution to obtain hGVs. Next, two rounds of centrifugally assisted flotation are followed by removal of the subnatant layer to ensure complete removal of native GvpC. For detailed information, see Lakshmanan et al. [30].

\section{Tissue-mimicking phantom 3D design and preparation}

Tissue-mimicking phantoms for imaging are prepared by melting $1 \%(\mathrm{w} / \mathrm{v})$ agarose gel in PBS and with $0.2 \%(\mathrm{w} / \mathrm{v})$ $\mathrm{AlO}_{3}$. We use a custom 3D-printed mold to create a 2-by-2 
grid of cylindrical wells with $2 \mathrm{~mm}$ diameter and $1 \mathrm{~mm}$ spacing between the outer radii in the bulk material. GVs are incubated at $42^{\circ} \mathrm{C}$ for 1 minute and then mixed in a $1: 1$ ratio with molten agarose (at $42^{\circ} \mathrm{C}$ ) for a final $\mathrm{GV}$ concentration corresponding to 2.25 OD500 nm and immediately loaded into the phantom. Wells not containing $\mathrm{GVs}$ are filled with plain $1 \%$ agar. The $\mathrm{AlO}_{3}$ concentration is chosen to match the scattering echogenicity of the GV well as measured by the contrast-to-noise ratio of the respective regions in a B-mode ultrasound image. The phantoms used for the angle ramp images contain stripped Ana GVs in the upper-left well. The phantom used for the voltage ramp images contains wild-type Ana GVs in the upper-left well and stripped Ana GVs in the upper-right well. All phantoms are imaged on top of an acoustic absorber material while immersed in PBS. Based on the elevation f-number of the probe, the elevation resolution (i.e., the thickness of the imaging plane) is $512 \mu \mathrm{m}$. The molarity of Ana GVs for a given OD value is $114 \mathrm{pM} / \mathrm{OD}$ [30]. Using these values and the dimensions of the $\mathrm{hGV}$ inclusion, we estimate that $2.47 \times 10^{5} \mathrm{GVs}$ contribute to each image, or roughly $200 \mathrm{GVs}$ for each pixel.

\section{Ultrasound acquisition sequence}

We use a Verasonics Vantage ultrasound system with a L22-14v probe (Verasonics Inc., Redmond, WA, USA) to implement the XAM and pAM imaging sequences. The probe is a linear array of 128 elements with a $0.10-\mathrm{mm}$ pitch, an 8-mm elevation focus, a $1.5-\mathrm{mm}$ elevation aperture, and a center frequency of $18.5 \mathrm{MHz}$ with $67 \%$ $-6 \mathrm{~dB}$ bandwidth. We apply a single-cycle transmit waveform at $15.625 \mathrm{MHz}$ to each active array element to ensure our fundamental frequency is divided 4 times with the 62.5$\mathrm{MHz}$ sampling rate of the system. To provide a reasonable tradeoff between the lateral field of view and the axial depth of field, we use an aperture of 65 elements for the xAM sequence (with the center element silenced to allow for a symmetric AM code). This allows for 64 ray lines per XAM image. The focus of the parabola used in the pAM sequence is set to $8 \mathrm{~mm}$ to match the probe's elevation focus. We use an aperture of 38 elements for the pAM sequence to maintain an f-number of 2 , but we limit the number of ray lines to 64 to match the XAM frames. To control for variation in pressure across different beam profiles, we select probe voltages for each $\mathrm{xAM}$ angle and for $\mathrm{pAM}$ that generate a CTR of $10 \mathrm{~dB}$ in the hGV inclusion of the phantoms. The voltages are given in Table I in Appendix B.

We collect raw rf data from our acquisitions and implement a custom real-time image reconstruction pipeline, including a beamforming algorithm suited to the unique requirements of xAM. To reduce noise during live imaging while saving system memory, we apply a first-order infinite impulse response (IIR) filter to successive frames of rf data, according to the following difference equation:

$$
y[n]=\alpha y[n-1]+(1-\alpha) x[n],
$$

where $n$ is the frame index, $\alpha$ is the persistence coefficient, $x$ is the unfiltered rf data, and $y$ is the output of the filter. All rf data reported are acquired with $\alpha=0.9$ except for the in vivo $\mathrm{pAM}$ image, which is acquired with $\alpha=0.7$ to avoid blurring due to motion.

\section{XAM beamforming}

The novel cross-propagation paradigm necessitates adjustments to conventional beamforming for image reconstruction; a particularity of this method is that xAM image lines are not formed along the propagation direction of the ultrasonic waves but along the line on which the two cross-propagating plane waves intersect. The linear array transmission configuration and directivity [25] can be modeled as in Fig. 7(a). The distance from either angled wavefront to the point $\left(x_{b}, z\right)$ and the return trip distance of the echo received by array element $x_{n}$ are, respectively,

$$
\begin{gathered}
d_{T X}\left(\theta, x_{b}, z\right)=\left(x_{b}-x_{1}\right) p \sin \theta+z \cos \theta \\
d_{R X}\left(x_{b}, x_{n}, z\right)=\sqrt{\left(x_{n}-x_{b}\right)^{2} p^{2}+z^{2}} .
\end{gathered}
$$

Hence, the two-way travel time to element $x_{n}$ is

$$
\begin{aligned}
\tau_{\mathrm{T} / R \rightarrow x_{n}}= & \frac{1}{c}\left[\left(x_{b}-x_{1}\right) p \sin \theta+z \cos \theta\right] \\
& +\frac{1}{c} \sqrt{\left(x_{n}-x_{b}\right)^{2} p^{2}+z^{2}},
\end{aligned}
$$

whereas the observed arrival time of this echo on the bisector element is

$$
\tau_{\mathrm{T} / R \rightarrow x_{b}}=\frac{1}{c}\left[\left(x_{b}-x_{1}\right) p \sin \theta+z \cos \theta+z\right] .
$$

We can then derive the depth of the echo signal from its arrival time on the bisector as

$$
z=\frac{c \tau_{\mathrm{T} / R \rightarrow x_{b}}-\left(x_{b}-x_{1}\right) p \sin \theta}{\cos \theta+1},
$$

and, finally, we can obtain the time delay to apply to the received signal for dynamic focusing:

$$
\begin{aligned}
\delta\left(x_{n}, z\right) & =\tau_{\mathrm{T} / R \rightarrow x_{n}}-\tau_{\mathrm{T} / R \rightarrow x_{b}} \\
& =\frac{1}{c}\left[\sqrt{\left(x_{n}-x_{b}\right)^{2} p^{2}+z^{2}}-z\right]
\end{aligned}
$$

These beamforming equations are valid in the region over which the waves are cross propagating. The depth of field [Eq. (1)] to which this region extends is given by $\left(x_{n}-x_{b}\right) \cot \theta$. 
(a)

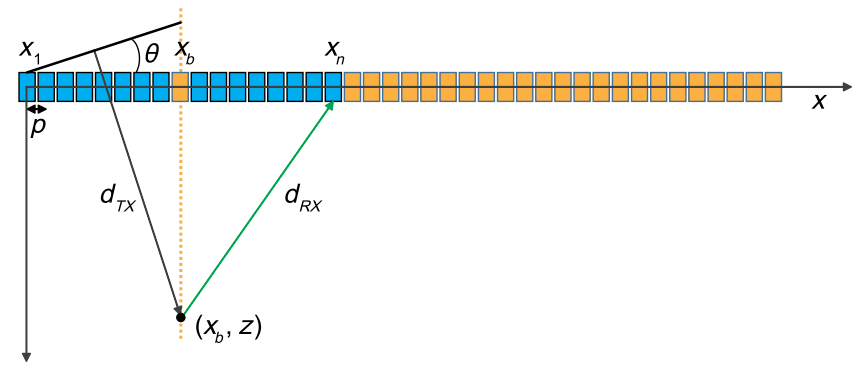

(b)

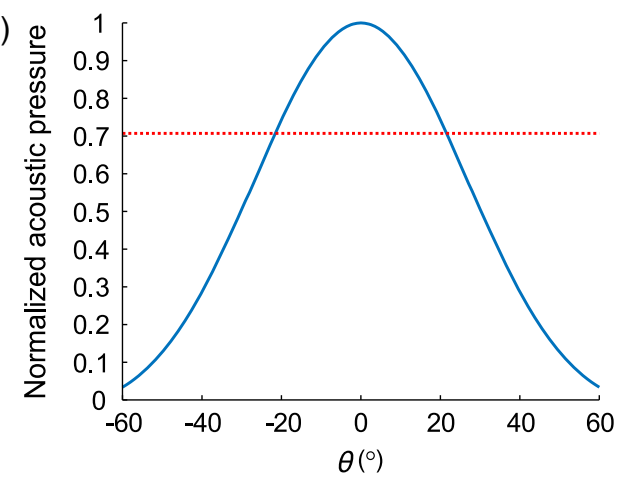

FIG. 7. Linear array aperture geometry and directivity. (a) Ultrasound imaging linear array configuration. Here, $\theta$ is the crosspropagation angle, $p$ the pitch of the linear transducer array, $x_{1}$ the first element of the active aperture (blue elements), $x_{b}$ the element along the aperture, and $x_{n}$ an arbitrary element along the array. Note that $d_{t x}$ is the distance from the planar wavefront to a point along the bisector, and $d_{r x}$ is the return distance to the array. Silent elements are labeled in orange. (b) Directivity of an individual element of the linear transducer array $(p=0.1 \mathrm{~mm}, f=15.6 \mathrm{MHz})$. The red dotted line indicates the $-3 \mathrm{~dB}$ acoustic pressure level.

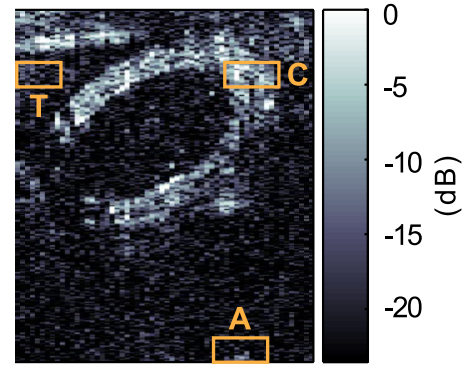

FIG. 8. Tissue (T), contrast (C), and artifact (A) regions of interest used for the ratios displayed in Fig. 6.

\section{In vivo ultrasound imaging}

The in vivo experiment is performed on a C57BL/6J male mouse (Jackson Laboratory) under a protocol approved by the Institutional Animal Care and Use Committee of the California Institute of Technology. No randomization or blinding are necessary in this study. Ultrasound imaging is performed as follows: The mouse is anaesthetized with $2 \%-3 \%$ isoflurane, depilated over the imaged region, and imaged using an L22-14v transducer with the pulse sequence described above. For imaging of GVs in the gastrointestinal tract, the mouse is placed in a supine position, with the ultrasound transducer positioned on the lower abdomen, transverse to the colon. Prior to imaging, wild-type and stripped Ana GVs are mixed in a $1: 1$ ratio with $42^{\circ} \mathrm{C} 4 \%$ agarose-PBS for a final $\mathrm{GV}$ $\mathrm{OD}_{500 \mathrm{~nm}}$ equal to 2.25. An 8-gauge needle is filled with the mixture of agarose and stripped Ana GVs. Before it solidifies, a 14-gauge needle is placed inside the 8-gauge needle to form a hollow lumen within the gel. After the agarose-GV mixture solidifies at room temperature for $10 \mathrm{~min}$, the 14-gauge needle is removed. The hollow lumen is then filled with the agarose mixture containing the wildtype Ana GVs. After it solidifies, the complete cylindrical agarose gel is injected into the colon of the mouse with a PBS back-filled syringe. Additional PBS is then injected into the colon to remove air bubbles in the vicinity of the gel.

\section{APPENDIX B: SUPPLEMENTARY DATA \\ 1. Supersonic cross-propagating plane-wave intersection}

Interestingly, as both plane waves cross propagate, local coordinates of each wave-front interact with their axisymmetric counterpart, but contrary to copropagating plane waves, it is a transient interaction. The plane-wave intersection velocity $c_{X}$ is given by

$$
c_{X}=c_{0} / \cos \theta,
$$

where $c_{0}$ is the speed of sound in the propagation medium. It can readily be seen from Eq. (2) that the plane-wave intersection velocity $c_{X}$ is supersonic for $\theta>0$ as reported by $\mathrm{Lu}$ and Greenleaf [28], and seen in

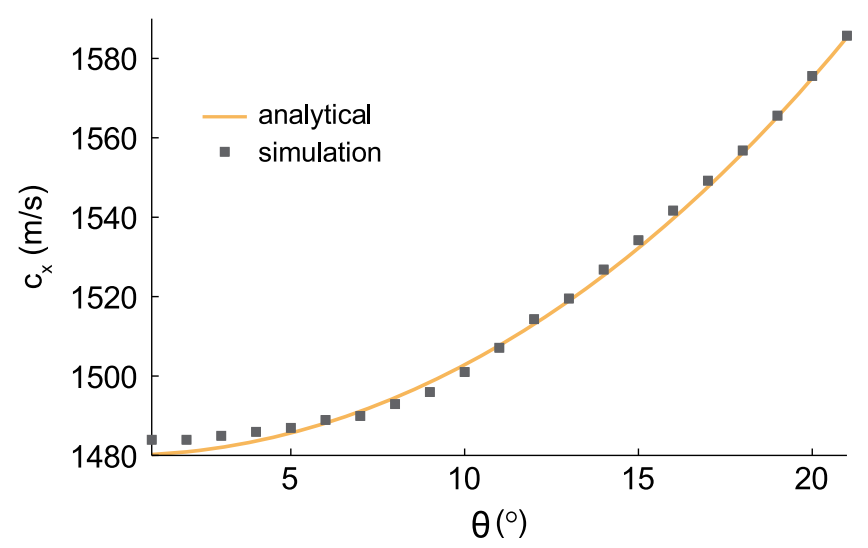

FIG. 9. Analytical and simulated cross-propagating plane-wave intersection velocity as a function of $\theta$. 
(a)

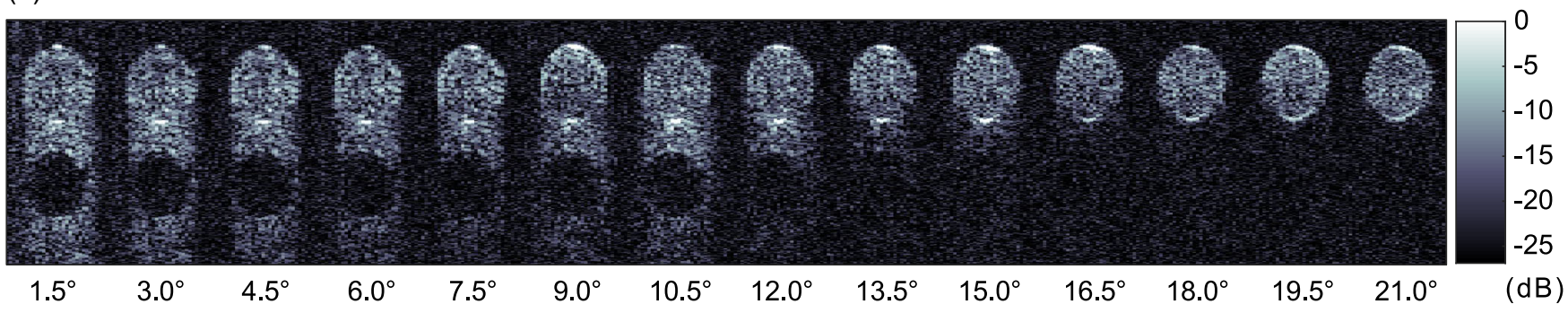

(b)

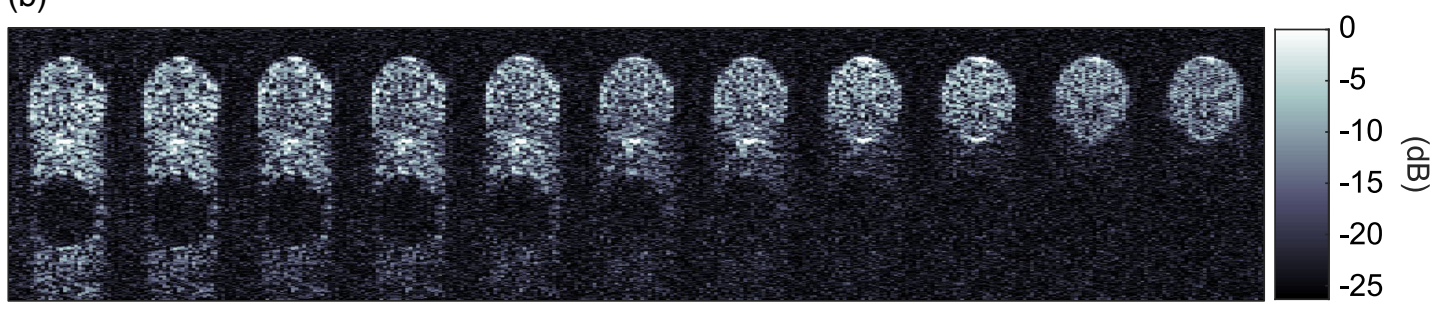

$15^{\circ}-6^{\circ} \quad 3^{\circ}-75^{\circ} \quad 45^{\circ}-9^{\circ} \quad 6^{\circ}-105^{\circ} \quad 7.5^{\circ}-12^{\circ} \quad 9^{\circ}-13.5^{\circ} 10.5^{\circ}-15^{\circ} 12^{\circ}-16.5^{\circ} 13.5^{\circ}-18^{\circ} 15^{\circ}-19.5^{\circ} 16.5^{\circ}-21^{\circ}$

(c)

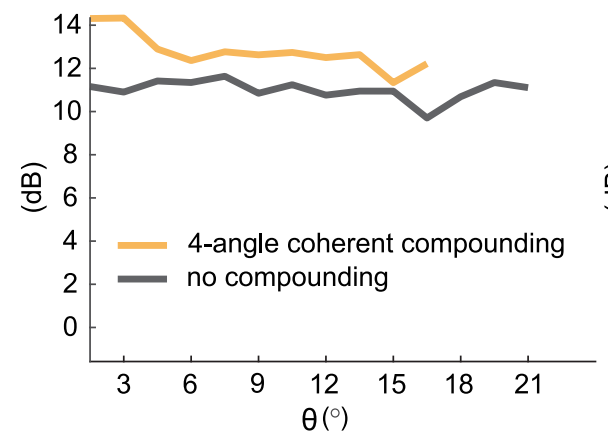

(d)

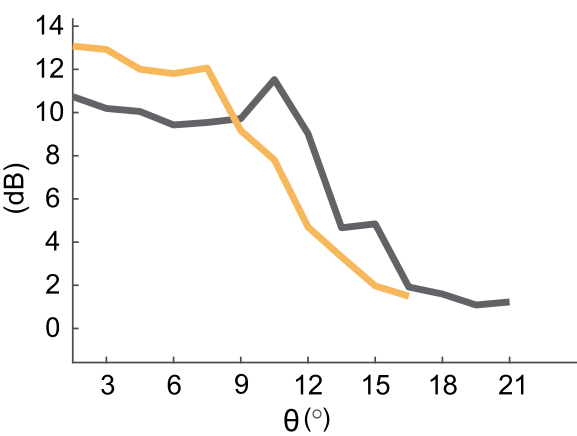

(e)

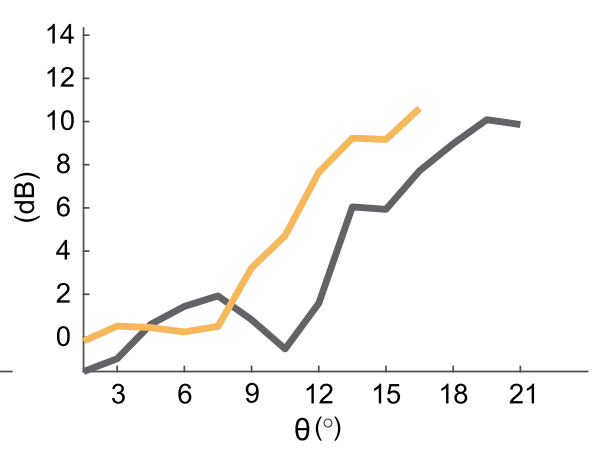

FIG. 10. Comparison of coherently compounded and single-acquisition xAM images of the hGV inclusion in the tissuemimicking phantom reported in Fig. 5(a). (a) A set of xAM images from the experiment depicted in Fig. 5. (b) The same images with coherent compounding applied to successive sets of four acquisitions. (c) Contrast-to-tissue ratio of single-acquisition xAM compared with coherently compounded data as a function of angle. (d) Artifact-to-tissue ratio. (e) Contrast-to-artifact ratio. Here, $n=6$. Error bars are not shown for ease of comparison.

Fig. 9. The third transmission event of the xAM sequence [Fig. 2(c)] corresponds to a finite-aperture forwardpropagating $\mathrm{X}$-wave solution of the homogeneous and isotropic wave equation with the form $f\left(x, z-c_{X} t\right)$ [28], where $f$ represents a scalar function (e.g., the acoustic pressure pulse) of space and time, although in our case Xwave pressure distribution is constant along its branches.

\section{Coherent compounding}

We test the effect of coherent compounding [35] of the rf data from multiple XAM acquisitions with different angles. Because of the difference in interaction velocity for different angles, the rf data from individual acquisitions are first aligned with the peak of the average autocorrelation function of the individual beamformed ray lines composing the images. The best results are achieved by compounding four adjacent angles. This improved the average CTR by $1.7 \mathrm{~dB}$ and the peak CAR by $0.5 \mathrm{~dB}$ (Fig. 10).

\section{Voltage-pressure table}

The phantom images reported in Fig. 5 are acquired using the following voltage table. They ensure a $10 \mathrm{~dB}$ CTR across images and therefore enable for the comparison of the artifact intensity across cross-propagation angles.

TABLE I. The pAM (shaded) and xAM (white) input transducer voltages generating a 10-dB CTR at $4 \mathrm{~mm}$.

\begin{tabular}{|c|c|c|c|c|c|c|c|c|c|c|c|c|c|c|c|}
\hline$\theta\left(^{\circ}\right)$ & pAM & 1.5 & 3.0 & 4.5 & 6.0 & 7.5 & 9.0 & 10.5 & 12.0 & 13.5 & 15.0 & 16.5 & 18.0 & 19.5 & 21.0 \\
\hline$V_{4 \mathrm{~mm}}$ & 1.9 & 4.6 & 4.3 & 3.7 & 3.3 & 3.4 & 3.8 & 3.9 & 4.2 & 4.1 & 4.5 & 4.5 & 4.7 & 5.0 & 5.5 \\
\hline
\end{tabular}


[1] R. Y. Tsien, The Green Fluorescent Protein, Annu. Rev. Biochem. 67, 509 (1998).

[2] V. Ntziachristos, Going Deeper than Microscopy: The Optical Imaging Frontier in Biology, Nat. Methods 7, 603 (2010).

[3] R. W. Bourdeau, A. Lee-Gosselin, A. Lakshmanan, A. Farhadi, S. R. Kumar, S. P. Nety, and M. G. Shapiro, Acoustic Reporter Genes for Noninvasive Imaging of Microorganisms in Mammalian Hosts, Nature (London) 553, 86 (2018).

[4] M. G. Shapiro, P. W. Goodwill, A. Neogy, M. Yin, F. S. Foster, D. V. Schaffer, and S. M. Conolly, Biogenic Gas Nanostructures as Ultrasonic Molecular Reporters, Nat. Nanotechnol. 9, 311 (2014).

[5] K. Ferrara, R. Pollard, and M. Borden, Ultrasound Microbubble Contrast Agents: Fundamentals and Application to Gene and Drug Delivery, Annu. Rev. Biomed. Eng. 9, 415 (2007).

[6] T. Faez, M. Emmer, K. Kooiman, M. Versluis, A. van der Steen, and N. de Jong, 20 Years of Ultrasound Contrast Agent Modeling, IEEE Trans. Ultrason. Ferroelectr. Freq. Control 60, 7 (2013).

[7] G. A. Brock-Fisher, M. D. Poland, and P. G. Rafter (Google Patents, 1996).

[8] D. H. Simpson, C. T. Chin, and P. N. Burns, Pulse Inversion Doppler: A New Method for Detecting Nonlinear Echoes from Microbubble Contrast Agents, IEEE Trans. Ultrason. Ferroelectr. Freq. Control 46, 372 (1999).

[9] R. Hansen and B. A. Angelsen, Contrast Imaging by Nonoverlapping Dual Frequency Band Transmit Pulse Complexes, IEEE Trans. Ultrason. Ferroelectr. Freq. Control 58, 290 (2011).

[10] D. Maresca, G. Renaud, G. van Soest, X. Li, Q. Zhou, K. K. Shung, N. de Jong, and A. F. van der Steen, ContrastEnhanced Intravascular Ultrasound Pulse Sequences for Bandwidth-Limited Transducers, Ultrasound Med. Biol. 39, 706 (2013).

[11] G. Renaud, J. G. Bosch, G. L. Ten Kate, V. Shamdasani, R. Entrekin, N. de Jong, and A. F. van der Steen, Counter-Propagating Wave Interaction for ContrastEnhanced Ultrasound Imaging, Phys. Med. Biol. 57, L9 (2012).

[12] A. E. Walsby, Gas Vesicles, Microbiol. Rev. 58, 94 (1994).

[13] D. Maresca et al., Biomolecular Ultrasound and Sonogenetics, Annu. Rev. Chem. Biomol. Eng. 9, 229 (2018).

[14] A. Lakshmanan, A. Farhadi, S. P. Nety, A. Lee-Gosselin, R. W. Bourdeau, D. Maresca, and M. G. Shapiro, Molecular Engineering of Acoustic Protein Nanostructures, ACS Nano 10, 7314 (2016).

[15] D. Maresca, A. Lakshmanan, A. Lee-Gosselin, J. M. Melis, Y.L. Ni, R. W. Bourdeau, D. M. Kochmann, and M. G. Shapiro, Nonlinear Ultrasound Imaging of Nanoscale Acoustic Biomolecules, Appl. Phys. Lett. 110, 073704 (2017).

[16] J. Wu and J. Tong, Measurements of the Nonlinearity Parameter B/A of Contrast Agents, Ultrasound Med. Biol. 24, 153 (1998).

[17] M. Emmer, H. J. Vos, D. E. Goertz, A. van Wamel, M. Versluis, and N. de Jong, Pressure-Dependent Attenuation and Scattering of Phospholipid-Coated Microbubbles at Low Acoustic Pressures, Ultrasound Med. Biol. 35, 102 (2009).

[18] M.-X. Tang and R. J. Eckersley, Frequency and Pressure Dependent Attenuation and Scattering by Microbubbles, Ultrasound Med. Biol. 33, 164 (2007).

[19] G. L. ten Kate et al., Far-Wall Pseudoenhancement During Contrast-Enhanced Ultrasound of the Carotid Arteries: Clinical Description and In Vitro Reproduction, Ultrasound Med. Biol. 38, 593 (2012).

[20] S. I. Aanonsen, T. Barkve, J. N. Tjo/tta, and S. Tjo/tta, Distortion and Harmonic Generation in the Nearfield of a Finite Amplitude Sound Beam, J. Acoust. Soc. Am. 75, 749 (1984).

[21] V. Gusev, H. Bailliet, P. Lotton, and M. Bruneau, Interaction of Counterpropagating Acoustic Waves in Media with Nonlinear Dissipation and in Hysteretic Media, Wave Motion 29, 211 (1999).

[22] G. Renaud, J. G. Bosch, A. F. van der Steen, and N. de Jong, Increasing Specificity of Contrast-Enhanced Ultrasound Imaging Using the Interaction of Quasi Counter-Propagating Wavefronts: A Proof of Concept, IEEE Trans. Ultrason. Ferroelectr. Freq. Control 62, 1768 (2015).

[23] M. Pasovic, M. Danilouchkine, T. Faez, P. L. van Neer, C. Cachard, A. F. van der Steen, O. Basset, and N. de Jong, Second Harmonic Inversion for Ultrasound Contrast Harmonic Imaging, Phys. Med. Biol. 56, 3163 (2011).

[24] B. E. Treeby, J. Jaros, A. P. Rendell, and B. T. Cox, Modeling Nonlinear Ultrasound Propagation in Heterogeneous Media with Power Law Absorption Using a k-space Pseudospectral Method, J. Acoust. Soc. Am. 131, 4324 (2012).

[25] A. Selfridge, G. Kino, and B. Khuri-Yakub, A Theory for the Radiation Pattern of a Narrow-Strip Acoustic Transducer, Appl. Phys. Lett. 37, 35 (1980).

[26] M. F. Hamilton and J. A. TenCate, Sum and Difference Frequency Generation Due to Noncollinear Wave Interaction in a Rectangular Duct, J. Acoust. Soc. Am. 81, 1703 (1987).

[27] M. F. Hamilton and D. T. Blackstock, On the Coefficient of Nonlinearity $\beta$ in Nonlinear Acoustics, J. Acoust. Soc. Am. 83, 74 (1988).

[28] J. Y. Lu and J. F. Greenleaf, Nondiffracting X Waves-Exact Solutions to Free-Space Scalar Wave Equation and Their Finite Aperture Realizations, IEEE Trans. Ultrason. Ferroelectr. Freq. Control 39, 19 (1992).

[29] J.-Y. Lu and J. F. Greenleaf, in IEEE 1991 Ultrasonics Symposium (IEEE, 1991), Vol. 1152, pp. 1155-1159, https://ieeexplore.ieee.org/document/234298/citations.

[30] A. Lakshmanan et al., Preparation of Biogenic Gas Vesicle Nanostructures for Use as Contrast Agents for Ultrasound and MRI, Nat. Protoc. 12, 2050 (2017).

[31] K. Wang, W. Sun, C. T. Richie, B. K. Harvey, E. Betzig, and N. Ji, Direct Wavefront Sensing for High-Resolution In Vivo Imaging in Scattering Tissue, Nat. Commun. 6, 7276 (2015).

[32] M. Imbault, A. Faccinetto, B.-F. Osmanski, A. Tissier, T. Deffieux, J.-L. Gennisson, V. Vilgrain, and M. Tanter, 
Robust Sound Speed Estimation for Ultrasound-Based Hepatic Steatosis Assessment, Phys. Med. Biol. 62, 3582 (2017).

[33] M. Tanter and M. Fink, Ultrafast Imaging in Biomedical Ultrasound, IEEE Trans. Ultrason. Ferroelectr. Freq. Control 61, 102 (2014).

[34] J. Provost, C. Papadacci, J. E. Arango, M. Imbault, M. Fink, J.-L. Gennisson, M. Tanter, and M. Pernot, 3D Ultrafast
Ultrasound Imaging In Vivo, Phys. Med. Biol. 59, L1 (2014).

[35] G. Montaldo, M. Tanter, J. Bercoff, N. Benech, and M. Fink, Coherent Plane-Wave Compounding for Very High Frame Rate Ultrasonography and Transient Elastography, IEEE Trans. Ultrason. Ferroelectr. Freq. Control 56, 489 (2009). 